\title{
A Family of Generalized Stirling Numbers of the First Kind
}

\author{
Beih S. El-Desouky¹, Nabela A. El-Bedwehy' ${ }^{2}$, Abdelfattah Mustafa', Fatma M. Abdel Menem² \\ ${ }^{1}$ Mathematics Department, Faculty of Science, Mansoura University, Mansoura, Egypt \\ ${ }^{2}$ Mathematics Department, Faculty of Science, Damietta University, Damietta, Egypt \\ Email: $\underline{\text { b desouky@yahoo.com }}$
}

Received 4 March 2014; revised 4 April 2014; accepted 11 April 2014

Copyright @ 2014 by authors and Scientific Research Publishing Inc.

This work is licensed under the Creative Commons Attribution International License (CC BY).

http://creativecommons.org/licenses/by/4.0/

CC) (i) Open Access

\begin{abstract}
A modified approach via differential operator is given to derive a new family of generalized Stirling numbers of the first kind. This approach gives us an extension of the techniques given by El-Desouky [1] and Gould [2]. Some new combinatorial identities and many relations between different types of Stirling numbers are found. Furthermore, some interesting special cases of the generalized Stirling numbers of the first kind are deduced. Also, a connection between these numbers and the generalized harmonic numbers is derived. Finally, some applications in coherent states and matrix representation of some results obtained are given.
\end{abstract}

\section{Keywords}

Stirling Numbers, Comtet Numbers, Creation, Annihilation, Differential Operator, Maple Program

\section{Introduction}

Gould [2] proved that

$$
\left(\mathrm{e}^{x} D\right)^{n}=\mathrm{e}^{n x} \sum_{k=1}^{n}(-1)^{n-k} s(n, k) D^{k}=\mathrm{e}^{n x} \sum_{k=1}^{n} s_{1}(n, k) D^{k}, D=D_{x}=\frac{\mathrm{d}}{\mathrm{d} x},
$$

where $s(n, k)$ and $s_{1}(n, k)$ are the usual Stirling numbers and the singles Stirling numbers of the first kind, respectively, defined by

$$
\begin{gathered}
(x)_{n}=\sum_{k=0}^{n} s(n, k) x^{k}, s(n, 0)=\delta_{n, 0} \text { and } s(n, k)=0 \text { for } k>n . \\
\langle x\rangle_{n}=\sum_{k=0}^{n} s_{1}(n, k) x^{k}, s_{1}(n, 0)=\delta_{n, 0} \text { and } s_{1}(n, k)=0 \text { for } k>n,
\end{gathered}
$$


where $(x)_{n}=x(x-1) \cdots(x-n+1)$ and $\langle x\rangle=x(x+1) \cdots(x+n-1)$.

These numbers satisfy the recurrence relations

$$
\begin{aligned}
& s(n+1, k)=s(n, k-1)-n s(n, k), \\
& s_{1}(n+1, k)=s_{1}(n, k-1)+n s_{1}(n, k) .
\end{aligned}
$$

EL-Desouky [1] defined the generalized Stirling numbers of the first kind $s(k ; \overline{\boldsymbol{r}}, \overline{\boldsymbol{s}})$, called $(\overline{\boldsymbol{r}}, \overline{\boldsymbol{s}})$-Stirling numbers of the first kind by

$$
\mathrm{e}^{r_{n} x} D^{s_{n}} \cdots \mathrm{e}^{r_{2} x} D^{s_{2}} \mathrm{e}^{r_{1} x} D^{s_{1}}=\mathrm{e}^{\left(\sum_{l=1}^{n} r_{1}\right) x} \sum_{k=s_{1}}^{s_{1}+s_{2}+\cdots+s_{n}} s(k ; \overline{\boldsymbol{r}}, \overline{\boldsymbol{s}}) D^{k},
$$

$s(k ; \overline{\boldsymbol{r}}, \overline{\boldsymbol{s}})=0$ for $k<s_{1}$ or $k>\sum_{j=1}^{n} s_{j}$ and $s(0 ; \overline{\boldsymbol{r}}, \boldsymbol{s})=1$, where $\overline{\boldsymbol{r}}:=\left(r_{1}, r_{2}, \cdots, r_{n}\right)$ is a sequence of real numbers and $\overline{\boldsymbol{s}}:=\left(s_{1}, s_{2}, \cdots, s_{n}\right)$ is a sequence of nonnegative integers.

Equation (6) is equivalent to

$$
\mathrm{e}^{r_{n} a^{+}} a^{s_{n}} \cdots \mathrm{e}^{r_{2} a^{+}} a^{s_{2}} \mathrm{e}^{r_{1} a^{+}} a^{s_{1}}=\mathrm{e}^{\left(\sum_{l=1}^{n} r_{l}\right) a^{+} s_{1}+s_{2}+\cdots+s_{n}} \sum_{k=s_{1}} s(k ; \overline{\boldsymbol{r}}, \overline{\boldsymbol{s}}) a^{k},
$$

where $a^{+}$and $a$ are boson creation and annihilation operators, respectively, and satisfy the commutation relation $\left[a^{+}, a\right]=1$.

The numbers $s(k ; \overline{\boldsymbol{r}}, \overline{\boldsymbol{s}})$ satisfy the recurrence relation

$$
s\left(k ; \overline{\boldsymbol{r}} \oplus r_{n+1}, \overline{\boldsymbol{s}} \oplus s_{n+1}\right)=\sum_{i=0}^{s_{n+1}}\left(\begin{array}{l}
s_{n+1} \\
i
\end{array}\right)\left(\sum_{j=1}^{n} r_{j}\right)^{s_{n+1}-i} s(k-i ; \overline{\boldsymbol{r}}, \overline{\boldsymbol{s}}),
$$

with the notations $\overline{\boldsymbol{r}} \oplus r_{n+1}=\left(r_{1}, r_{2}, \cdots, r_{n+1}\right)$ and $\overline{\boldsymbol{s}} \oplus s_{n+1}=\left(s_{1}, s_{2}, \cdots, s_{n+1}\right)$.

The numbers $s(k ; \overline{\boldsymbol{r}}, \overline{\boldsymbol{s}})$ have the explicit formula

$$
s(k ; \overline{\boldsymbol{r}}, \overline{\boldsymbol{s}})=\sum_{\sigma_{n-1}=\beta_{n}-k, j \geq 0} \prod_{l=1}^{n-1}\left(\begin{array}{l}
s_{l+1} \\
i_{l}
\end{array}\right)\left(\sum_{j=1}^{l} r_{j}\right)^{i_{l}},
$$

where $\sigma_{n}=\sum_{j=0}^{n} i_{j}$, with $i_{0}=0$ and $\beta_{n}=\sum_{j=1}^{n} s_{j}$.

Moreover El-Desouky [1] derived many special cases and some applications. For the proofs and more details, see [1].

The generalized falling factorial of $x$ associated with the sequence $\bar{\alpha}:=\left(\alpha_{0}, \alpha_{1}, \cdots, \alpha_{n-1}\right)$ of order $n$, where $\alpha_{0}, \alpha_{1}, \cdots, \alpha_{n-1}$ are real numbers, is defined by $(x, \bar{\alpha})_{n}=\prod_{i=0}^{n-1}\left(x-\alpha_{i}\right),(x, \bar{\alpha})_{0}=1$.

Comtet [3] [4] and [5] defined $s_{\bar{\alpha}}(n, k)$ the generalized Stirling numbers of the first kind, which are called Comtet numbers, by

$$
(x, \bar{\alpha})_{n}=\sum_{k=0}^{n} s_{\bar{\alpha}}(n, k) x^{k}
$$

These numbers satisfy the recurrence relation

$$
s_{\bar{\alpha}}(n, k)=s_{\bar{\alpha}}(n-1, k-1)-\alpha_{n-1} s_{\bar{\alpha}}(n-1, k) .
$$

El-Desouky and Cakic [6] defined $s_{\bar{\alpha}}(n, k ; \bar{s})$, the generalized Comtet numbers by

$$
\prod_{j=0}^{n-1}\left(x^{\alpha_{j}} \delta x^{-\alpha_{j}}\right)^{s_{j}}=\prod_{j=0}^{n-1}\left(\delta-\alpha_{j}\right)^{s_{j}}=\sum_{k=0}^{|s|} s_{\bar{\alpha}}(n, k ; \tilde{\mathbf{s}}) \delta^{k},
$$

where $|\boldsymbol{s}|=\sum_{j=0}^{n-1} s_{j}, s_{\bar{\alpha}}(n, k ; s)=0$ for $k>|\boldsymbol{s}|, \mathrm{s}_{\bar{\alpha}}(n, 0 ; \tilde{\boldsymbol{s}})=(-1)^{|s|} \prod_{j=0}^{n-1}\left(\alpha_{j}\right)^{s_{j}}$ and $\tilde{\boldsymbol{s}}=\left(s_{0}, s_{1}, \cdots, s_{n}\right)$.

For more details on generalized Stirling numbers via differential operators, see [7]-[10] and [11]. 
The paper is organized as follows:

In Section 2, using the differential operator $\left(\mathrm{e}^{r_{n} x} D^{s_{n}} \mathrm{e}^{r_{n} x}\right) \cdots\left(\mathrm{e}^{r_{2} x} D^{s_{2}} \mathrm{e}^{r_{2} x}\right)\left(\mathrm{e}^{r_{1} x} D^{s_{1}} \mathrm{e}^{r_{1} x}\right)$ we define a new family of generalized Stirling numbers of the first kind, denoted by $s(k ; \overline{\boldsymbol{r}}, \overline{\boldsymbol{s}})$. A recurrence relation and an explicit formula of these numbers are derived. In Section 3, some interesting special cases are discussed. Moreover some new combinatorial identities and a connection between $s(n, k ; r, s)$ and the generalized harmonic numbers $O_{n}^{(i)}$ are given. In Section 4, some applications in coherent states and matrix representation of some results obtained are given. Section 5 is devoted to the conclusion, which handles the main results derived throughout this work. Finally, a computer program is written using Maple and executed for calculating the generalized Stirling numbers of the first kind and some special cases, see Appendix.

\section{Main Results}

Let $\overline{\boldsymbol{r}}:=\left(r_{1}, r_{2}, \cdots, r_{n}\right)$ be a sequence of real numbers and $\overline{\boldsymbol{s}}:=\left(s_{1}, s_{2}, \cdots, s_{n}\right)$ be a sequence of nonnegative integers.

\section{Definition 2.1}

The generalized Stirlng numbers $\boldsymbol{s}(k ; \overline{\boldsymbol{r}}, \overline{\boldsymbol{s}})$ are defined by

$$
\left(\mathrm{e}^{r_{n} x} D^{s_{n}} \mathrm{e}^{r_{n} x}\right) \cdots\left(\mathrm{e}^{r_{2} x} D^{s_{2}} \mathrm{e}^{r_{2} x}\right)\left(\mathrm{e}^{r_{1} x} D^{s_{1}} \mathrm{e}^{r_{1} x}\right)=\mathrm{e}^{\left(2 \sum_{l=1}^{n} r^{r_{1}}\right)} \sum_{k=0}^{\beta_{n}} \boldsymbol{s}(k ; \overline{\boldsymbol{r}}, \overline{\boldsymbol{s}}) D^{k},
$$

where $\beta_{n}=\sum_{j=1}^{n} s_{j}, \boldsymbol{s}(k ; \overline{\boldsymbol{r}}, \overline{\boldsymbol{s}})=0$ for $k>\beta_{n}$ and $\boldsymbol{s}(0 ; \overline{\boldsymbol{r}}, \overline{\boldsymbol{s}})=1$.

Equation (13) is equivalent to

$$
\left(\mathrm{e}^{r_{n} a^{+}} a^{s_{n}} \mathrm{e}^{r_{n} a^{+}}\right) \cdots\left(\mathrm{e}^{r_{2} a^{+}} a^{s_{2}} \mathrm{e}^{r_{2} a^{+}}\right)\left(\mathrm{e}^{r_{1} a^{+}} a^{s_{1}} \mathrm{e}^{r_{1} a^{+}}\right)=\mathrm{e}^{\left(2 \sum_{l=1}^{n} r_{1}\right) a^{+}} \sum_{k=0}^{\beta} \boldsymbol{s}(k ; \overline{\boldsymbol{r}}, \overline{\boldsymbol{s}}) a^{k} .
$$

\section{Theorem 2.1}

The numbers $\boldsymbol{s}(k ; \overline{\boldsymbol{r}}, \overline{\boldsymbol{s}})$ satisfy the recurrence relation

$$
\boldsymbol{s}\left(k ; \overline{\boldsymbol{r}} \oplus r_{n+1}, \overline{\mathbf{s}} \oplus s_{n+1}\right)=\sum_{i=0}^{s_{n+1}}\left(\begin{array}{c}
s_{n+1} \\
i
\end{array}\right)\left(2 \sum_{j=1}^{n} r_{j}+r_{n+1}\right)^{s_{n+1}-i} \boldsymbol{s}(k-i ; \overline{\boldsymbol{r}}, \overline{\boldsymbol{s}}),
$$

with the notations $\overline{\boldsymbol{r}} \oplus r_{n+1}:=\left(r_{1}, r_{2}, \cdots, r_{n+1}\right)$ and $\overline{\boldsymbol{s}} \oplus s_{n+1}:=\left(s_{1}, s_{2}, \cdots, s_{n+1}\right)$.

\section{Proof}

$$
\begin{aligned}
\mathrm{e}^{\left(2 \sum_{j=1}^{n+1} r_{j}\right) x} \sum_{k=0}^{\beta_{n+1}} \boldsymbol{s}(k ; \overline{\boldsymbol{r}}, \overline{\boldsymbol{s}}) D^{k} & =\left(\mathrm{e}^{r_{n+1} x} D^{s_{n+1}} \mathrm{e}^{r_{n+1} x}\right)\left(\mathrm{e}^{\left(2 \sum_{j=1}^{n} r_{j}\right) x} \sum_{k=0}^{\beta_{n}} \boldsymbol{s}(k ; \overline{\boldsymbol{r}}, \overline{\boldsymbol{s}}) D^{k}\right) \\
& =\mathrm{e}^{r_{n+1} x} D^{s_{n+1}}\left(\mathrm{e}^{\left(2 \sum_{j=1}^{n} r_{j}+r_{n+1}\right) x} \sum_{k=0}^{\beta_{n}} \boldsymbol{s}(k ; \overline{\boldsymbol{r}}, \overline{\boldsymbol{s}}) D^{k}\right) \\
& =\mathrm{e}^{\left(2 \sum_{j=1}^{n} r_{j}\right) x} \sum_{m=0}^{\beta_{n}}\left(D+\left(2 \sum_{j=1}^{n} r_{j}+r_{n+1}\right)\right)^{s_{n+1}} \boldsymbol{s}(m ; \overline{\boldsymbol{r}}, \overline{\boldsymbol{s}}) D^{m} \\
& =\mathrm{e}^{\left(2 \sum_{j=1}^{n} r_{j}\right) \times} \sum_{m=0}^{\beta_{n}} \sum_{i=0}^{s_{n+1}}\left(\begin{array}{c}
s_{n+1} \\
i
\end{array}\right)\left(2 \sum_{j=1}^{n} r_{j}+r_{n+1}\right)^{s_{n+1}-i} \boldsymbol{s}(m ; \overline{\boldsymbol{r}}, \overline{\boldsymbol{s}}) D^{m+i} \\
& =\mathrm{e}^{\left(2 \sum_{j=1}^{n} r_{j}\right) \times} \sum_{k=0}^{\beta_{n+1}} \sum_{i=0}^{s_{n+1}}\left(\begin{array}{c}
s_{n+1} \\
i
\end{array}\right)\left(2 \sum_{j=1}^{n} r_{j}+r_{n+1}\right)^{s_{n+1}-i} \boldsymbol{s}(k-i ; \overline{\boldsymbol{r}}, \overline{\boldsymbol{s}}) D^{k}
\end{aligned}
$$

Equating the coefficients of $D^{k}$ on both sides yields (15).

\section{Theorem 2.2}

The numbers $\boldsymbol{s}(k ; \overline{\boldsymbol{r}}, \overline{\boldsymbol{s}})$ have the explicit formula

$$
\boldsymbol{s}(k ; \overline{\boldsymbol{r}}, \overline{\boldsymbol{s}})=\sum_{\sigma_{n}=\beta_{n}-k, j \geq 0} \prod_{l=1}^{n}\left(\begin{array}{c}
s_{i} \\
i
\end{array}\right)\left(2\left(\sum_{j=0}^{l-1} r_{j}\right)+r_{l}\right)^{i_{l}} \text {, where } \sigma_{n}=\sum_{j=1}^{n} i_{j}, r_{0}=0 \text {. }
$$




\section{Proof}

$$
\begin{aligned}
& \mathrm{e}^{r_{1} x} D^{s_{1}} \mathrm{e}^{r_{1} x}=\mathrm{e}^{r_{1} x} \mathrm{e}^{r_{1} x}\left(D+r_{1} I\right)^{s_{1}}=\mathrm{e}^{2 r_{1} x} \sum_{i_{1}=0}^{s_{1}}\left(\begin{array}{c}
s_{1} \\
i_{1}
\end{array}\right)\left(r_{1}\right)^{i_{1}} D^{s_{1}-i_{1}}, \\
&\left(\mathrm{e}^{r_{2} x} D^{s_{2}} \mathrm{e}^{r_{2} x}\right)\left(\mathrm{e}^{r_{1} x} D^{s_{1}} \mathrm{e}^{r_{1} x}\right)=\mathrm{e}^{r_{2} x} D^{s_{2}} \mathrm{e}^{r_{2} x}\left(\mathrm{e}^{2 r_{1} x} \sum_{i_{1}=0}^{s_{1}}\left(\begin{array}{c}
s_{1} \\
i_{1}
\end{array}\right)\left(r_{1}\right)^{i_{1}} D^{s_{1}-i_{1}}\right)=\mathrm{e}^{r_{2} x} D^{s_{2}} \mathrm{e}^{\left(2 r_{1}+r_{2}\right) x} \sum_{i_{1}=0}^{s_{1}}\left(\begin{array}{c}
s_{1} \\
i_{1}
\end{array}\right)\left(r_{1}\right)^{i_{1}} D^{s_{1}-i_{1}} \\
&=\mathrm{e}^{r_{2} x} \mathrm{e}^{\left(2 r_{1}+r_{2}\right) x} \sum_{i_{1}=0}^{s_{1}}\left(\begin{array}{c}
s_{1} \\
i_{1}
\end{array}\right)\left(r_{1}\right)^{i_{1}}\left(D+\left(2 r_{1}+r_{2}\right) I\right)^{s_{2}} D^{s_{1}-i_{1}} \\
&=\mathrm{e}^{2\left(r_{1}+r_{2}\right) x} \sum_{i_{1}=0}^{s_{1}} \sum_{i_{2}=0}^{s_{2}}\left(\begin{array}{c}
s_{1} \\
i_{1}
\end{array}\right)\left(\begin{array}{l}
s_{2} \\
i_{2}
\end{array}\right)\left(r_{1}\right)^{i_{1}}\left(2 r_{1}+r_{2}\right)^{i_{2}} D^{s_{1}+s_{2}-i_{1}-i_{2}},
\end{aligned}
$$

thus, by iteration, we get

$$
\begin{aligned}
& \left(\mathrm{e}^{r_{n} x} D^{s_{n}} \mathrm{e}^{r_{n} x}\right) \cdots\left(\mathrm{e}^{r_{2} x} D^{s_{2}} \mathrm{e}^{r_{2} x}\right)\left(\mathrm{e}^{r_{1} x} D^{s_{1}} \mathrm{e}^{r_{1} x}\right) \\
= & \mathrm{e}^{2\left(\sum_{l=1}^{n} r_{l}\right) x} \sum_{i_{1}=0}^{s_{1}} \sum_{i_{2}=0}^{s_{2}} \cdots \sum_{i_{n}=0}^{s_{n}} \prod_{j=1}^{n}\left(\begin{array}{l}
s_{j} \\
i_{j}
\end{array}\right)\left(2\left(\sum_{l=1}^{j-1} r_{l}\right)+r_{j}\right)^{i_{j}} D^{\sum_{j=1}^{n} s_{j}-\sum_{j=1}^{n} i_{j}}, r_{0}=0 .
\end{aligned}
$$

Setting $\sum_{j=1}^{n} s_{j}-\sum_{j=1}^{n} i_{j}=\beta_{n}-\sigma_{n}=k$, we obtain

$$
\left(\mathrm{e}^{r_{n} x} D^{s_{n}} \mathrm{e}^{r_{n} x}\right) \cdots\left(\mathrm{e}^{r_{2} x} D^{s_{2}} \mathrm{e}^{r_{2} x}\right)\left(\mathrm{e}^{r_{1} x} D^{s_{1}} \mathrm{e}^{r_{1} x}\right)=\mathrm{e}^{\left(2 \sum_{l=1}^{n} r_{l}\right) x} \sum_{k=0}^{\beta_{n}} \sum_{\sigma_{n}=\beta_{n}-k, j \geq 0} \prod_{j=1}^{n}\left(\begin{array}{l}
s_{j} \\
i_{j}
\end{array}\right)\left(2\left(\sum_{l=1}^{j-1} r_{l}\right)+r_{j}\right)^{i_{j}} D^{k} .
$$

Comparing (13) and (18) yields (16).

\section{Special Cases}

Setting $r_{i}=r$ and $s_{i}=s, i=1, \cdots, n$ in (13), we have the following definition.

\section{Definition 3.1}

For any real number $r$ and nonnegative integer $s$, let the numbers $\boldsymbol{s}(n, k ; r, s)$, be defined by

$$
\left(\mathrm{e}^{r x} D^{s} \mathrm{e}^{r x}\right)^{n}=\mathrm{e}^{(2 n r) x} \sum_{k=0}^{n s} \boldsymbol{s}(n, k ; r, s) D^{k},
$$

where $\boldsymbol{s}(0,0 ; r, s)=1$ and $\boldsymbol{s}(n, k ; r, s)=0$ for $k>n s$.

Equation (19) is equivalent to

$$
\left(\mathrm{e}^{r a^{+}} a^{s} \mathrm{e}^{r a^{+}}\right)^{n}=\mathrm{e}^{(2 n r) a^{+}} \sum_{k=0}^{n s} \boldsymbol{s}(n, k ; r, s) a^{k}
$$

\section{Corollary 3.1}

The numbers $\boldsymbol{s}(n, k ; r, s)$ satisfy the recurrence relation

$$
\boldsymbol{s}(n+1, k ; r, s)=\sum_{i=0}^{s}\left(\begin{array}{l}
s \\
i
\end{array}\right)((2 n+1) r)^{s-i} \boldsymbol{s}(n, k-i ; r, s) .
$$

\section{Proof}

The proof follows directly from Equation (15) by setting $r_{i}=r$ and $s_{i}=s, i=1,2, \cdots, n$.

\section{Corollary 3.2}

The numbers $\boldsymbol{s}(n, k ; r, s)$ have the explicit formula

$$
\boldsymbol{s}(n, k ; r, s)=r^{n s-k} \sum_{\sigma_{n}=n s-k, i_{j} \geq 0}\left(\prod_{j=1}^{n}\left(\begin{array}{l}
s \\
i_{j}
\end{array}\right)(2 j-1)^{i_{j}}\right) .
$$

\section{Proof}

By substituting $r_{i}=r$ and $s_{i}=s, i=1,2, \cdots, n$ in Equation (17), yields 


$$
\left(\mathrm{e}^{r x} D^{s} \mathrm{e}^{r x}\right)^{n}=\mathrm{e}^{(2 n r) x} \sum_{0 \leq i_{1}, \cdots, i_{n} \leq s}\left(\prod_{j=1}^{n}\left(\begin{array}{l}
s \\
i_{j}
\end{array}\right)(2 j-1)^{i_{j}} r^{\sigma_{n}}\right) D^{n s-\sigma_{n}},
$$

then setting $n s-\sigma_{n}=k$ we have

$$
\left(\mathrm{e}^{r x} D^{s} \mathrm{e}^{r x}\right)^{n}=\mathrm{e}^{(2 n r) x} \sum_{k=0}^{n s} r^{n s-k} \sum_{\sigma_{n}=n s-k, i_{j} \geq 0}\left(\prod_{j=1}^{n}\left(\begin{array}{l}
s \\
i_{j}
\end{array}\right)(2 j-1)^{i_{j}}\right) D^{k},
$$

hence comparing Equations (19) and (23) we obtain Equation (22).

Furthermore we handle the following special cases.

i) If $r=1$, then we have

Definition 3.2

$$
\left(\mathrm{e}^{x} D^{s} \mathrm{e}^{x}\right)^{n}=\mathrm{e}^{(2 n) x} \sum_{k=0}^{n s} \boldsymbol{s}(n, k ; 1, s) D^{k},
$$

where $\boldsymbol{s}(0,0 ; 1, s)=1$ and $\boldsymbol{s}(n, k ; 1, s)=0$ for $k>n s$.

Corollary 3.3

The numbers $\boldsymbol{s}(n, k ; 1, s)$ satisfy the recurrence relation

$$
\boldsymbol{s}(n+1, k ; 1, s)=\sum_{i=0}^{s}\left(\begin{array}{l}
s \\
i
\end{array}\right)(2 n+1)^{s-i} \boldsymbol{s}(n, k-i ; 1, s) .
$$

Proof:

The proof follows directly from Equation (21) by setting $r=1$.

\section{Corollary 3.4}

The numbers $\boldsymbol{s}(n, k ; 1, s)$ have the explicit formula

$$
\boldsymbol{s}(n, k ; 1, s)=\sum_{\sigma_{n}=n s-k, i_{j} \geq 0}\left[\prod_{j=1}^{n}\left(\begin{array}{l}
s \\
i_{j}
\end{array}\right)(2 j-1)^{i_{j}}\right] .
$$

\section{Proof}

The proof follows directly from Equation (22) by setting $r=1$.

ii) If $s=1$, then we have

\section{Definition 3.3}

The numbers $\boldsymbol{s}(n, k ; r, 1)$ are defined by

$$
\left(\mathrm{e}^{r x} D \mathrm{e}^{r x}\right)^{n}=\mathrm{e}^{(2 n r) x} \sum_{k=0}^{n} \boldsymbol{s}(n, k ; r, 1) D^{k},
$$

where $\boldsymbol{s}(n, 0 ; r, 1)=\delta_{n, 0}$ and $\boldsymbol{s}(n, k ; r, 1)=0$ for $k>n$.

\section{Corollary 3.5}

The numbers $\boldsymbol{s}(n, k ; r, 1)$ satisfy the triangular recurrence relation

$$
\boldsymbol{s}(n, k ; r, 1)=\boldsymbol{s}(n-1, k-1 ; r, 1)+(2 n-1) r \boldsymbol{s}(n-1, k ; r, 1) .
$$

\section{Proof}

The proof follows easily from (22) by setting $s=1$.

\section{Corollary 3.6}

The numbers $\boldsymbol{s}(n, k ; r, 1)$ have the following explicit formula

$$
\boldsymbol{s}(n, k ; r, 1)=r^{n-k} \sum_{\sigma_{n}=n-k, i_{j} \in\{0,1\}} \prod_{j=1}^{n}(2 j-1)^{i_{j}} .
$$

\section{Proof}

The proof follows from (22) by setting $s=1$.

Also, using the recurrence relation (28) we can find the following explicit formula. 


\section{Theorem 3.1}

The numbers $\boldsymbol{s}(n, k ; r, 1)$ have the following explicit expression

$$
\boldsymbol{s}(n, k ; r, 1)=\sum_{i_{1}+\cdots+i_{n}=k, i_{j} \in\{0,1\}}\left(\begin{array}{c}
i_{1}+r \\
1-i_{1}
\end{array}\right)\left(\begin{array}{c}
i_{2}+3 r \\
1-i_{2}
\end{array}\right) \cdots\left(\begin{array}{c}
i_{n}+(2 n-1) r \\
1-i_{n}
\end{array}\right) .
$$

\section{Proof}

$$
\text { For } k=0, \boldsymbol{s}(n, 0 ; r, 1)=(r)(3 r)(5 r) \cdots((2 n-1) r)=r^{n}(1.2 .3 \cdots(2 n-1)) .
$$

For $i_{n} \in\{0,1\}$, we get

$$
\begin{aligned}
\boldsymbol{s}(n, k ; r, 1) & \sum_{i_{1}+\cdots+i_{n-1}=k-0, i_{j} \in\{0,1\}}\left(\begin{array}{c}
i_{1}+r \\
1-i_{1}
\end{array}\right)\left(\begin{array}{c}
i_{2}+3 r \\
1-i_{2}
\end{array}\right) \ldots\left(\begin{array}{c}
i_{n-1}+(2 n-3) r \\
1-i_{n-1}
\end{array}\right) \cdot(2 n-1) r \\
& +\sum_{i_{1}+\cdots+i_{n-1}=k-1, i_{j} \in\{0,1\}}\left(\begin{array}{c}
i_{1}+r \\
1-i_{1}
\end{array}\right)\left(\begin{array}{c}
i_{2}+3 r \\
1-i_{2}
\end{array}\right) \ldots\left(\begin{array}{c}
i_{n-1}+(2 n-3) r \\
1-i_{n-1}
\end{array}\right) \cdot 1 \\
= & \boldsymbol{s}(n-1, k-1 ; r, 1)+(2 n-1) r \boldsymbol{s}(n-1, k ; r, 1) .
\end{aligned}
$$

That is the same recurrence relation (28) for the numbers $\boldsymbol{s}(n, k ; r, 1)$. This completes the proof. iii) If $r=1$ and $s=1$, then we have

\section{Definition 3.4}

The numbers $\boldsymbol{s}(n, k):=\boldsymbol{s}(n, k ; 1,1)$ are defined by

$$
\left(\mathrm{e}^{x} D \mathrm{e}^{x}\right)^{n}=\mathrm{e}^{2 n x} \sum_{k=0}^{n} \boldsymbol{s}(n, k) D^{k},
$$

where $\boldsymbol{s}(n, 0)=\delta_{n, 0}$ and $\boldsymbol{s}(n, k)=0$ for $k>n$.

Equation (31) is equivalent to

$$
\left(\mathrm{e}^{a^{+}} D \mathrm{e}^{a^{+}}\right)^{n}=\mathrm{e}^{2 n a^{+}} \sum_{k=0}^{n} \boldsymbol{s}(n, k) a^{k} .
$$

\section{Corollary 3.7}

The numbers $\boldsymbol{s}(n, k)$ satisfy the triangular recurrence relation

$$
\boldsymbol{s}(n+1, k)=\boldsymbol{s}(n, k-1)+(2 n+1) \boldsymbol{s}(n, k) .
$$

\section{Proof}

The proof follows by setting $r=1$ in Equation (28).

\section{Corollary 3.8}

The numbers $\boldsymbol{s}(n, k)$ have the explicit formula

$$
\boldsymbol{s}(n, k)=\sum_{\sigma_{n}=n-k, i_{j} \in\{0,1\}} \prod_{j=1}^{n}(2 j-1)^{i_{j}} .
$$

\section{Proof}

The proof follows by setting $r=1$ in Equation (29).

Moreover $\boldsymbol{s}(n, k)$ have the following explicit formula.

\section{Corollary 3.9}

The numbers $\boldsymbol{s}(n, k)$ have the following explicit expression

$$
\boldsymbol{s}(n, k)=\sum_{i_{1}+\cdots+i_{n}=k, i_{j} \in\{0,1\}}\left(\begin{array}{c}
i_{1}+1 \\
1-i_{1}
\end{array}\right)\left(\begin{array}{c}
i_{2}+3 \\
1-i_{2}
\end{array}\right)\left(\begin{array}{c}
i_{2}+5 \\
1-i_{3}
\end{array}\right) \ldots\left(\begin{array}{c}
i_{n}+(2 n-1) \\
1-i_{n}
\end{array}\right) .
$$

\section{Proof}

The proof follows by setting $r=1$ in (30). 
From Equations (29) and (30) (also from Equations (34) and (35)) we have the combinatorial identities

$$
\begin{array}{r}
\sum_{i_{1}+\cdots+i_{n}=k, i_{j} \in\{0,1\}}\left(\begin{array}{c}
i_{1}+r \\
1-i_{1}
\end{array}\right)\left(\begin{array}{c}
i_{2}+3 r \\
1-i_{2}
\end{array}\right) \ldots\left(\begin{array}{c}
i_{n}+(2 n-1) r \\
1-i_{n}
\end{array}\right)=r^{n-k} \sum_{\sigma_{n}=n-k, i_{j} \in\{0,1\}} \prod_{j=1}^{n}(2 j-1)^{i_{j}} . \\
\sum_{i_{1}+\cdots+i_{n}=k, i_{j} \in\{0,1\}}\left(\begin{array}{c}
i_{1}+1 \\
1-i_{1}
\end{array}\right)\left(\begin{array}{c}
i_{2}+3 \\
1-i_{2}
\end{array}\right) \ldots\left(\begin{array}{c}
i_{n}+(2 n-1) \\
1-i_{n}
\end{array}\right)=\sum_{\sigma_{n}=n-k, i_{j} \in\{0,1\}} \prod_{j=1}^{n}(2 j-1)^{i_{j}} .
\end{array}
$$

From Equations (29) and (34) we obtain that

$$
\boldsymbol{s}(n, k ; r, 1)=r^{n-k} \boldsymbol{s}(n, k) .
$$

\section{Remark 3.1}

Operating with both sides of Equation (13) on the exponential function $e^{l x}$, we get

$$
\left(l+r_{1}\right)^{s_{1}}\left(l+2 r_{1}+r_{2}\right)^{s_{2}}+\cdots+\left(l+2 r_{1}+\cdots+2 r_{n-1}+r_{n}\right)^{s_{n}}=\sum_{k=s}^{\beta_{n}} \boldsymbol{s}(k ; \overline{\boldsymbol{r}}, \overline{\boldsymbol{s}}) l^{k} .
$$

Therefore, since a nonzero polynomial can have only a finite set of zeros, we have

$$
\prod_{j=0}^{n-1}\left(2 \sum_{m=0}^{j} r_{m}+r_{j+1}+x\right)^{s_{j+1}}=\sum_{k=0}^{\beta_{n}} \boldsymbol{s}(k ; \overline{\boldsymbol{r}}, \overline{\boldsymbol{s}}) x^{k}, r_{0}=0 .
$$

If $x=1$, we obtain

$$
\sum_{k=0}^{\beta_{n}} \boldsymbol{s}(k ; \overline{\boldsymbol{r}}, \overline{\boldsymbol{s}})=\prod_{j=0}^{n-1}\left(1+2 \sum_{m=0}^{j} r_{m}+r_{j+1}\right)^{s_{j+1}} .
$$

\section{Remark 3.2}

From relation (39), by replacing $s_{j}$ with $s_{j-1}$, and relation (18) we conclude that

$$
\boldsymbol{s}(k ; \overline{\boldsymbol{r}}, \tilde{\boldsymbol{s}})=s_{\bar{\alpha}}(n, k ; \tilde{\boldsymbol{s}}) \text {, where } \alpha_{i}=-\left(2 \sum_{m=0}^{i} r_{m}+r_{i+1}\right), i=0,1, \cdots, n-1 .
$$

This gives us a connection between $\boldsymbol{s}(k ; \overline{\boldsymbol{r}}, \overline{\boldsymbol{s}})$ and $s_{\bar{\alpha}}(n, k ; \tilde{\boldsymbol{s}})$, the generalized Comtet numbers, see [6]. Setting $r_{i}=r$ and $s_{i}=s, i=1, \cdots, n$, in (39), we get

$$
\prod_{j=0}^{n-1}((2 j+1) r+x)^{s}=\sum_{k=0}^{n s} \boldsymbol{s}(n, k ; r, s) x^{k}
$$

hence, we have $s(n, k ; r, s)=s_{\bar{\alpha}}(n, k ; s)$, where $\alpha_{i}=-(2 i+1) r, i=0,1, \cdots, n-1$, see [6].

If $x=1$, then

$$
\sum_{k=0}^{n s} \boldsymbol{s}(n, k ; r, s)=\prod_{j=0}^{n-1}((2 j+1) r+1)^{s}, n \geq 1 .
$$

Next we discuss the following special cases of (42) and (43):

i) If $r=1$, then

$$
\left.\begin{array}{l}
\prod_{j=0}^{n-1}((2 j+1)+x)^{s}=\sum_{k=0}^{n s} \boldsymbol{s}(n, k ; 1, s) x^{k}, \\
\sum_{k=0}^{n s} \boldsymbol{s}(n, k ; 1, s)=\prod_{j=0}^{n-1}((2 j+1)+1)^{s}, n \geq 1,
\end{array}\right\}
$$

hence we have $\boldsymbol{s}(n, k ; 1, s)=s_{\bar{\alpha}}(n, k)$, the generalized Comtet numbers, where $\alpha_{i}=-(2 i+1)$, $i=0,1, \cdots, n-1$, see [6].

ii) If $s=1$, then we have 


$$
\left.\begin{array}{l}
\prod_{j=0}^{n-1}((2 j+1) r+x)=\sum_{k=0}^{n} \boldsymbol{s}(n, k ; r, 1) x^{k}, \\
\sum_{k=0}^{n} \boldsymbol{s}(n, k ; r, 1)=\prod_{j=0}^{n-1}((2 j+1) r+1), n \geq 1 .
\end{array}\right\}
$$

hence we obtain $\boldsymbol{s}(n, k ; r, s)=s_{\bar{\alpha}}(n, k)$, Comtet numbers, where $\alpha_{i}=-(2 i+1) r, i=0,1, \cdots, n-1$, see [3] and [4].

For example if $n=3, r=2$ and $s=2$ in (43) we have

$$
\sum_{k=0}^{6} \boldsymbol{s}(3, k ; 2,2)=\prod_{j=0}^{2}(4 j+3)^{2} .
$$

Using Table 2,

L.H.S. of $(46)=s(3,0 ; 2,2)+s(3,1 ; 2,2)+s(3,2 ; 2,2)+s(3,3 ; 2,2)+s(3,4 ; 2,2)+s(3,5 ; 2,2)+s(3,6 ; 2,2)=14400$ $+22080+12784+3552+508+36+1=53361$.

R.H.S. of $(46)=\prod_{j=0}^{2}(4 j+3)^{2}=\left(3^{2}\right)\left(7^{2}\right)\left(11^{2}\right)=53361$.

This confirms (46) and hence (43).

Another example if $n=2, r=2$ and $s=3$ in (43) we have

$$
\sum_{k=0}^{6} \boldsymbol{s}(2, k ; 2,3)=\prod_{j=0}^{1}(4 j+3)^{3} .
$$

Using Table 3,

L.H.S. of $(47)=s(2,0 ; 2,3)+s(2,1 ; 2,3)+s(2,2 ; 2,3)+s(2,3 ; 2,3)+s(2,4 ; 2,3)+s(2,5 ; 2,3)+s(2,6 ; 2,3)=1728+$ $3456+2736+1088+228+24+1=9261$.

R.H.S. of $(46)=\prod_{j=0}^{1}(4 j+3)^{3}=\left(3^{3}\right)\left(7^{3}\right)=9261$.

This confirms (43).

iii) If $r=s=1$, then we get

$$
\left.\begin{array}{l}
\prod_{j=0}^{n-1}((2 j+1)+x)=\sum_{k=0}^{n} \boldsymbol{s}(n, k) x^{k}, \\
\sum_{k=0}^{n} \boldsymbol{s}(n, k)=\prod_{j=0}^{n-1} 2(j+1)=2^{n} n !, n \geq 1,
\end{array}\right\}
$$

hence we have $\boldsymbol{s}(n, k)=s_{\bar{\alpha}}(n, k)$, which is a special case of Comtet numbers, where $\alpha_{i}=-(2 i+1), i=0,1, \cdots, n-1$, see [3] and [4] and Table 1.

Setting $\mathrm{e}^{x}=t$, we have $D:=\mathrm{d} / \mathrm{d} x=t(\mathrm{~d} / \mathrm{d} t)=t D_{t}=\delta_{t}$, then substituting in (2.1) it becomes

$$
\left(t^{r_{n}} \delta_{t}^{s_{n}} t^{r_{n}}\right) \cdots\left(t^{r_{2}} \delta_{t}^{s_{2}} t^{r_{2}}\right)\left(t^{r_{1}} \delta_{t}^{s_{1}} t^{r_{1}}\right)=\mathrm{e}^{\left(2 \sum_{l=1}^{n} r_{l}\right) x} \sum_{k=0}^{\beta_{n}} \boldsymbol{s}(k ; \overline{\boldsymbol{r}}, \overline{\boldsymbol{s}}) \delta_{t}^{k} .
$$

Using, see [12],

$$
F(\delta)\left(x^{\alpha} f(x)\right)=x^{\alpha} F(\delta+\alpha) f(x)
$$

then Equation (49) yields

$$
\left(\delta_{t}+2 r_{1}+\cdots+2 r_{n-1}+r_{n}\right)^{s_{n}} \cdots\left(\delta_{t}+2 r_{2}+r_{1}\right)^{s_{2}}\left(\delta_{t}+r_{1}\right)^{s_{1}}=\sum_{k=0}^{\beta_{n}} \boldsymbol{s}(k ; \overline{\boldsymbol{r}}, \overline{\boldsymbol{s}}) \delta_{t}^{k} .
$$

Comparing this equation with Equation (4.1) in [6], we get

$$
\boldsymbol{s}(k ; \overline{\boldsymbol{r}}, \tilde{\boldsymbol{s}})=s_{\bar{\alpha}}(n, k ; \tilde{\boldsymbol{s}}),
$$

where $\alpha_{i}=-\left(2 \sum_{m=0}^{i} r_{m}+r_{i+1}\right), i=0,1, \cdots, n-1$ and $s_{\bar{\alpha}}(n, k ; \tilde{\boldsymbol{s}})$, are the generalized Comtet numbers of the first 
kind.

Furthermore, using our notations, it is easy from Equation (4.4) in [6] and (41) to show that

$$
S(n, i ; \overline{\boldsymbol{\alpha}}, \tilde{\boldsymbol{s}})=\sum_{k=i}^{|s|} \boldsymbol{s}(n, k ; \overline{\boldsymbol{r}}, \tilde{\boldsymbol{s}}) S(k, i),|s|=s_{0}+s_{1}+\cdots+s_{n-1},
$$

where $\alpha_{i}=-\left(2 \sum_{m=0}^{i} r_{m}+r_{i+1}\right), i=0,1, \cdots, n-1$ and $S(n, k)$ are the Stirling numbers of the second kind.

Next, we find a connection between $s(n, k ; r, s)$ and the generalized harmonic numbers $O_{n}^{(i)}$ which are defined by, see [13] and [14],

$$
O_{n}^{(i)}=\sum_{j=1}^{n} \frac{1}{(2 j-1)^{i}}
$$

From (42), we have

$$
\begin{aligned}
& \sum_{k=0}^{n s} s(n, k ; r, s) x^{k} \\
& =\prod_{j=0}^{n-1}((2 j+1) r)^{s} \prod_{j=0}^{n-1}(1+x /(2 j+1) r)^{s}=\prod_{j=0}^{n-1}((2 j+1) r)^{s} \exp \left(\log \left(\prod_{j=0}^{n-1}(1+x /(2 j+1) r)^{s}\right)\right) \\
& =\prod_{j=0}^{n-1}((2 j+1) r)^{s} \exp \left(\sum_{j=0}^{n-1} s \log (1+x /(2 j+1) r)\right)=\prod_{j=0}^{n-1}((2 j+1) r)^{s} \exp \left(\sum_{i=1}^{\infty} s \sum_{j=0}^{n-1} \frac{(-1)^{i+1}}{((2 j+1) r)^{i}} \frac{x^{i}}{i}\right) \\
& =\prod_{j=0}^{n-1}((2 j+1) r)^{s} \exp \left(s \sum_{i=1}^{\infty} O_{n}^{(i)} \frac{(-1)^{i+1}}{r^{i} i} x^{i}\right)=\prod_{j=0}^{n-1}((2 j+1) r)^{s} \sum_{l=0}^{\infty}\left(s \sum_{i=1}^{\infty} O_{n}^{(i)} \frac{(-1)^{i+1}}{r^{i} i} x^{i}\right)^{l} / l ! \\
& =\prod_{j=0}^{n-1}((2 j+1) r)^{s} \sum_{l=0}^{\infty} \frac{s^{l}}{l !} \sum_{k=l}^{\infty} \sum_{i_{1}+\cdots+i_{l}=k}(-1)^{k+l} \frac{O_{n}^{\left(i_{1}\right)} \cdots O_{n}^{\left(i_{l}\right)}}{i_{1} \cdots i_{l} r^{k}} x^{k} \\
& =\prod_{j=0}^{n-1}((2 j+1) r)^{s} \sum_{l=0}^{\infty} \sum_{k=l}^{\infty} \frac{s^{l}}{l !} \sum_{i_{1}+\cdots+i_{l}=k}(-1)^{k+l} \frac{O_{n}^{\left(i_{1}\right)} \cdots O_{n}^{\left(i_{l}\right)}}{i_{1} \cdots i_{l} r^{k}} x^{k} .
\end{aligned}
$$

Equating the coefficients of $x^{k}$ on both sides, we obtain

$$
\boldsymbol{s}(n, k ; r, s)=r^{n s-k} \prod_{j=0}^{n-1}(2 j+1)^{s} \sum_{l=0}^{\infty} \frac{s^{l}}{l !} \sum_{k=l}^{\infty} \sum_{i_{1}+\cdots+i_{l}=k}(-1)^{k+l} \frac{O_{n}^{\left(i_{1}\right)} \cdots O_{n}^{\left(i_{l}\right)}}{i_{1} \cdots i_{l} r^{k}} .
$$

From (22) and (53), we have the combinatorial identity

$$
\sum_{\substack{\sigma_{n}=n s-k, i_{j} \geq 0}}\left[\prod_{j=1}^{n}\left(\begin{array}{l}
s \\
i_{j}
\end{array}\right)(2 j-1)^{i_{j}}\right]=\prod_{j=0}^{n-1}(2 j+1)^{s} \sum_{l=0}^{\infty} \frac{s^{l}}{l !} \sum_{k=l}^{\infty} \sum_{i_{1}+\cdots+i_{l}=k}(-1)^{k+l} \frac{O_{n}^{\left(i_{1}\right)} \cdots O_{n}^{\left(i_{l}\right)}}{i_{1} \cdots i_{l} r^{k}} .
$$

hence, setting $s=1$, we get the identity

$$
\sum_{\substack{\sigma_{n}=n-k, i_{j} \geq 0}}\left[\prod_{j=1}^{n}(2 j-1)^{i_{j}}\right]=\prod_{j=0}^{n-1}(2 j+1) \sum_{l=0}^{k} \sum_{i_{1}+\cdots+i_{l}=k}(-1)^{k+l} \frac{O_{n}^{\left(i_{1}\right)} \cdots O_{n}^{\left(i_{l}\right)}}{i_{1} \cdots i_{l} l !} .
$$

\section{Some Applications}

\subsection{Coherent State and Normal Ordering}

Coherent states play an important role in quantum mechanics especially in optics. The normally ordered form of the boson operator in which all the creation operators $a^{+}$stand to the left of the annihilation operators $a$. Using the properties of coherent states we can define and represent the generalized polynomial $\bar{P}_{r, s}(x)$ and generalized 
number $\bar{P}_{r, s}$ as follows.

\section{Definition 4.1}

The generalized polynomial $\bar{P}_{r, s}(x)$ is defined by

$$
\bar{P}_{r, s}(x)=\sum_{k=0}^{\beta_{n}} \boldsymbol{s}(k ; \overline{\boldsymbol{r}}, \overline{\boldsymbol{s}}) x^{k},
$$

and the generalized number $\bar{P}_{r, s}$

$$
\bar{P}_{r, s}=\bar{P}_{r, s}(1)=\sum_{k=0}^{\beta_{n}} \boldsymbol{s}(k ; \overline{\boldsymbol{r}}, \overline{\boldsymbol{s}}) .
$$

For convenience we apply the convention

$$
\boldsymbol{s}(k ; \overline{\boldsymbol{r}}, \overline{\boldsymbol{s}})=0 \text { for } k<0 \text { or } k>\beta_{n} .
$$

Now we come back to normal ordering. Using the properties of coherent states, see [7], the coherent state matrix element of the boson string yields the generalized polynomial $\bar{P}_{r, s}(x)$

$$
\begin{aligned}
& \left\langle z\left|\left(\mathrm{e}^{r_{n} a^{+}} a^{s_{n}} \mathrm{e}^{r_{n} a^{+}}\right) \cdots\left(\mathrm{e}^{r_{2} a^{+}} a^{s_{2}} \mathrm{e}^{r_{2} a^{+}}\right)\left(\mathrm{e}^{r_{1} a^{+}} a^{s_{1}} \mathrm{e}^{r_{1} a^{+}}\right)\right| z\right\rangle \\
& =\left\langle z\left|\mathrm{e}^{\left(2 \sum_{l=1}^{n} r^{\eta}\right) a^{+}} \sum_{k=0}^{\beta_{n}} \boldsymbol{s}(k ; \overline{\boldsymbol{r}}, \overline{\boldsymbol{s}}) a^{k}\right| z\right\rangle=\mathrm{e}^{\left(2 \sum_{l=1}^{n} r^{r}\right) z^{*}} \sum_{k=0}^{\beta_{n}} \boldsymbol{s}(k ; \overline{\boldsymbol{r}}, \overline{\boldsymbol{s}}) z^{k}\langle z \mid z\rangle=\mathrm{e}^{\left(2 \sum_{l=1}^{n} r^{r}\right) z^{*}} \sum_{k=0}^{n} \boldsymbol{s}(k, \overline{\boldsymbol{r}}, \overline{\boldsymbol{s}}) z^{k} \\
& =\mathrm{e}^{\left(2 \sum_{l=1}^{n} r_{l}\right) z^{*}} \bar{P}_{r, s}(z)=\mathrm{e}^{\left(2 \sum_{l=1}^{n} r_{l}\right) z^{*}} \bar{P}_{r, s}\left(\frac{|z|^{2}}{z^{*}}\right), \text { where } \beta_{n}=\sum_{j=1}^{n} s_{j} .
\end{aligned}
$$

\section{Definition 4.2}

We define the polynomial $\bar{P}(n, x)$ as

$$
\bar{P}(n, x)=\sum_{k=1}^{n} \boldsymbol{s}(n, k) x^{k},
$$

and the numbers

$$
\bar{P}(n)=\bar{P}(n, 1)=\sum_{k=1}^{n} \boldsymbol{s}(n, k) .
$$

For convenience we apply the conventions

$$
\boldsymbol{s}(n, 0)=\delta_{n, 0} \text { and } \boldsymbol{s}(n, k)=0 \text { for } k>n \text { and } \bar{P}(0)=\bar{P}(0, x)=1 .
$$

Similarly, using the properties of coherent states and (32) we have

$$
\begin{aligned}
\left\langle z\left|\left(\mathrm{e}^{a^{+}} a \mathrm{e}^{a^{+}}\right)\right| z\right\rangle & =\left\langle z\left(\mathrm{e}^{2 n a^{+}} \sum_{k=0}^{n} \boldsymbol{s}(n, k)\right) a^{k} \mid z\right\rangle \\
& =\mathrm{e}^{2 n z^{*}} \sum_{k=0}^{n} \boldsymbol{s}(n, k) z^{k}\langle z \mid z\rangle=\mathrm{e}^{2 n z^{*}} \sum_{k=0}^{n} \boldsymbol{s}(n, k) z^{k} \\
& =\mathrm{e}^{2 n z^{*}} \bar{P}(n, z)=\mathrm{e}^{2 n z^{*}} \bar{P}\left(n, \frac{|z|^{2}}{z^{*}}\right) .
\end{aligned}
$$

\subsection{Matrix Representation}

In this subsection we derive a matrix representation of some results obtained.

Let $s_{r}$ be $n \times n$ lower triangle matrix, where $s_{r}$ is the matrix whose entries are the numbers $s(n, k ; r, 1)$, i.e. $\boldsymbol{s}_{r}=[\boldsymbol{s}(i, j ; r, 1)]_{i, j \geq 0}$. Furthermore let $\boldsymbol{N}_{r}$ be an $n \times n$ lower triangle matrix defined by 
$\boldsymbol{N}_{r}=\left[\mathrm{e}^{2 i r x}(i, j ; r, 1)\right]_{i, j \geq 0}, \boldsymbol{M}_{r}$ is a diagonal matrix whose entries of the main diagonal are $\mathrm{e}^{2 i r x}, i=0,1, \cdots, n$, i.e. $\boldsymbol{M}_{\mathrm{r}}=\operatorname{diag}\left(\mathrm{e}^{0 r x}, \mathrm{e}^{2 r x}, \mathrm{e}^{4 r x}, \cdots, \mathrm{e}^{2 n r x}\right), \boldsymbol{R}_{\mathrm{r}}=\left(\left(\mathrm{e}^{r x} D \mathrm{e}^{r x}\right)^{0},\left(\mathrm{e}^{r x} D \mathrm{e}^{r x}\right)^{1}, \cdots,\left(\mathrm{e}^{r x} D \mathrm{e}^{r x}\right)^{n}\right)^{\mathrm{T}}$ and $D=\left(D^{0}, D^{1}, D^{2}, \cdots, D^{n}\right)^{\mathrm{T}}$.

Equation (27), may be represented in a matrix form as

$$
\boldsymbol{R}_{r}=\boldsymbol{N}_{r} \boldsymbol{D}=\boldsymbol{M}_{r} \boldsymbol{s}_{r} \boldsymbol{D},
$$

for example if $n=3$ then

$$
\begin{aligned}
{\left[\begin{array}{l}
\left(\mathrm{e}^{r x} D \mathrm{e}^{r x}\right)^{0} \\
\left(\mathrm{e}^{r x} D \mathrm{e}^{r x}\right)^{1} \\
\left(\mathrm{e}^{r x} D \mathrm{e}^{r x}\right)^{2} \\
\left(\mathrm{e}^{r x} D \mathrm{e}^{r x}\right)^{3}
\end{array}\right] } & {\left[\begin{array}{cccc}
\mathrm{e}^{0 r x} & 0 & 0 & 0 \\
r \mathrm{e}^{2 r x} & \mathrm{e}^{2 r x} & 0 & 0 \\
3 r^{2} \mathrm{e}^{4 r x} & 4 r \mathrm{e}^{4 r x} & \mathrm{e}^{4 r x} & 0 \\
15 r^{3} \mathrm{e}^{6 r x} & 23 r^{2} \mathrm{e}^{6 r x} & 9 r \mathrm{e}^{6 r x} & \mathrm{e}^{6 r x}
\end{array}\right]\left[\begin{array}{c}
D^{0} \\
D^{1} \\
D^{2} \\
D^{3}
\end{array}\right] } \\
& =\left[\begin{array}{cccc}
\mathrm{e}^{0 r x} & 0 & 0 & 0 \\
0 & \mathrm{e}^{2 r x} & 0 & 0 \\
0 & 0 & \mathrm{e}^{4 r x} & 0 \\
0 & 0 & 0 & \mathrm{e}^{6 r x}
\end{array}\right]\left[\begin{array}{cccc}
1 & 0 & 0 & 0 \\
r & 1 & 0 & 0 \\
3 r^{2} & 4 r & 1 & 0 \\
15 r^{3} & 23 r^{2} & 9 r & 1
\end{array}\right]\left[\begin{array}{c}
D^{0} \\
D^{1} \\
D^{2} \\
D^{3}
\end{array}\right],
\end{aligned}
$$

its inverse is given by

$$
\boldsymbol{D}=\boldsymbol{N}_{r}^{-1} \boldsymbol{R}_{r}=\boldsymbol{s}_{r}^{-1} \boldsymbol{M}_{r}^{-1} \boldsymbol{R}_{r} .
$$

Setting $r=1$ in (64), we get

$$
\boldsymbol{R}_{1}=\boldsymbol{N}_{1} \boldsymbol{D}=\boldsymbol{M}_{1} \boldsymbol{s}_{1} \boldsymbol{D},
$$

$$
\left[\begin{array}{l}
\left(\mathrm{e}^{x} D \mathrm{e}^{x}\right)^{0} \\
\left(\mathrm{e}^{x} D \mathrm{e}^{x}\right)^{1} \\
\left(\mathrm{e}^{x} D \mathrm{e}^{x}\right)^{2} \\
\left(\mathrm{e}^{x} D \mathrm{e}^{x}\right)^{3}
\end{array}\right]=\left[\begin{array}{cccc}
\mathrm{e}^{0 x} & 0 & 0 & 0 \\
\mathrm{e}^{2 x} & \mathrm{e}^{2 x} & 0 & 0 \\
3 \mathrm{e}^{4 x} & 4 \mathrm{e}^{4 x} & \mathrm{e}^{4 x} & 0 \\
15 \mathrm{e}^{6 x} & 23 \mathrm{e}^{6 x} & 9 \mathrm{e}^{6 x} & \mathrm{e}^{6 x}
\end{array}\right]\left[\begin{array}{c}
D^{0} \\
D^{1} \\
D^{2} \\
D^{3}
\end{array}\right]=\left[\begin{array}{cccc}
\mathrm{e}^{0 x} & 0 & 0 & 0 \\
0 & \mathrm{e}^{2 x} & 0 & 0 \\
0 & 0 & \mathrm{e}^{4 x} & 0 \\
0 & 0 & 0 & \mathrm{e}^{6 x}
\end{array}\right]\left[\begin{array}{cccc}
1 & 0 & 0 & 0 \\
1 & 1 & 0 & 0 \\
3 & 4 & 1 & 0 \\
15 & 23 & 9 & 1
\end{array}\right]\left[\begin{array}{c}
D^{0} \\
D^{1} \\
D^{2} \\
D^{3}
\end{array}\right],
$$

hence

$$
\boldsymbol{D}=\boldsymbol{N}_{1}^{-1} \boldsymbol{R}_{1}=\boldsymbol{s}_{1}^{-1} M_{1}^{-1} \boldsymbol{R}_{1}
$$

For $n=3$, we have

$$
\left[\begin{array}{l}
D^{0} \\
D^{1} \\
D^{2} \\
D^{3}
\end{array}\right]=\left[\begin{array}{cccc}
1 & 0 & 0 & 0 \\
-1 & \mathrm{e}^{-2 x} & 0 & 0 \\
1 & -4 \mathrm{e}^{-2 x} & \mathrm{e}^{-4 x} & 0 \\
-1 & 13 \mathrm{e}^{-2 x} & -9 \mathrm{e}^{-4 x} & \mathrm{e}^{-6 x}
\end{array}\right]\left[\begin{array}{l}
\left(\mathrm{e}^{x} D \mathrm{e}^{x}\right)^{0} \\
\left(\mathrm{e}^{x} D \mathrm{e}^{x}\right)^{1} \\
\left(\mathrm{e}^{x} D \mathrm{e}^{x}\right)^{2} \\
\left(\mathrm{e}^{x} D \mathrm{e}^{x}\right)^{3}
\end{array}\right]=\left[\begin{array}{cccc}
1 & 0 & 0 & 0 \\
-1 & 1 & 0 & 0 \\
1 & -4 & 1 & 0 \\
-1 & 13 & -9 & 1
\end{array}\right]\left[\begin{array}{cccc}
\mathrm{e}^{0 x} & 0 & 0 & 0 \\
0 & \mathrm{e}^{-2 x} & 0 & 0 \\
0 & 0 & \mathrm{e}^{-4 x} & 0 \\
0 & 0 & 0 & \mathrm{e}^{-6 x}
\end{array}\right]\left[\begin{array}{l}
\left(\mathrm{e}^{x} D \mathrm{e}^{x}\right)^{0} \\
\left(\mathrm{e}^{x} D \mathrm{e}^{x}\right)^{1} \\
\left(\mathrm{e}^{x} D \mathrm{e}^{x}\right)^{2} \\
\left(\mathrm{e}^{x} D \mathrm{e}^{x}\right)^{3}
\end{array}\right]
$$

\section{Conclusion}

In this article we investigated a new family of generalized Stirling numbers of the first kind. Recurrence relations and an explicit formula of these numbers are derived. Moreover some interesting special cases and new 
combinatorial identities are obtained. A connection between this family and the generalized harmonic numbers is given. Finally, some applications in coherent states and matrix representation of some results are obtained.

\section{References}

[1] El-Desouky, B.S. (2011) Generalized String Numbers of the First Kind: Modified Approach. Journal of Pure and Mathematics: Advances and Applications, 5, 43-59.

[2] Gould, H.W. (1964) The Operator $\left(a^{x} \Delta\right)^{n}$ and Stirling Numbers of the First Kind. The American Mathematical Monthly, 71, 850-858. http://dx.doi.org/10.2307/2312391

[3] Comtet, L. (1972) Nombres de stirling généraux et fonctions symétriques. Comptes Rendus de l'Académie des Sciences (Series A), 275, 747-750.

[4] Comtet, L. (1974) Advanced Combinatorics: The Art of Finite and Infinite Expiations. D. Reidel Publishing Company, Dordrecht, Holand. http://dx.doi.org/10.1007/978-94-010-2196-8

[5] Comtet, L. (1973) Une formule explicite pour les puissances successive de l'operateur derivation de Lie. Comptes Rendus de l'Académie des Sciences (Series A), 276, 165-168.

[6] El-Desouky, B.S. and Cakić, N.P. (2011) Generalized Higher Order Stirling Numbers. Mathematical and Computer Modelling, 54, 2848-2857. http://dx.doi.org/10.1016/j.mcm.2011.07.005

[7] Blasiak, P. (2005) Combinatorics of Boson Normal Ordering and Some Applications. PhD Thesis, University of Paris, Paris. http://arxiv.org/pdf/quant-ph/0507206.pdf

[8] Blasiak, P., Penson, K.A. and Solomon, A.I. (2003) The General Boson Normal Ordering Problem. Physics Letters A, 309, 198-205. http://dx.doi.org/10.1016/S0375-9601(03)00194-4

[9] Cakic, N.P. (1980) On Some Combinatorial Identities. Applicable Analysis and Discrete Mathematics, 678-715, 91-94.

[10] Carlitz, L. (1932) On Arrays of Numbers. American Journal of Mathematics, 54, 739-752. http://dx.doi.org/10.2307/2371100

[11] El-Desouky, B.S., Cakic, N.P. and Mansour, T. (2010) Modified Approach to Generalized Stirling Numbers via Differential Operators. Applied Mathematics Letters, 23, 115-120. http://dx.doi.org/10.1016/j.aml.2009.08.018

[12] Viskov, O.V. and Srivastava, H.M. (1994) New Approaches to Certain Identities Involving Differential Operators. Journal of Mathematical Analysis and Applications, 186, 1-10. http://dx.doi.org/10.1006/jmaa.1994.1281

[13] Macdonald, I.G. (1979) Symmetric Functions and Hall Polynomials. Clarendon (Oxford University) Press, Oxford, London and New York.

[14] Choi, J. and Srivastava, H.M. (2011) Some Summation Formulas Involving Harmonic Numbers and Generalized Harmonic Numbers. Mathematical and Computer Modelling, 54, 2220-2234. http://dx.doi.org/10.1016/j.mcm.2011.05.032. 


\section{Appendix}

Tables of $\boldsymbol{s}(n, k ; r, s)$ calculated using Maple, for some values of $n, k, r$ and $s$ :

Table 1. $0 \leq n, k \leq 4, r=s=1$.

\begin{tabular}{|c|c|c|c|c|c|}
\hline 1 & 0 & 0 & 0 & 0 & 1 \\
\hline 1 & 1 & 0 & 0 & 0 & 2 \\
\hline 3 & 4 & 1 & 0 & 0 & 8 \\
\hline 15 & 23 & 9 & 1 & 0 & 48 \\
\hline 105 & 176 & 86 & 16 & 1 & 384 \\
\hline
\end{tabular}

Table 2. $0 \leq n, k \leq 4, r=s=2$.

\begin{tabular}{|c|c|c|c|c|c|c|c|c|c|}
\hline 1 & 0 & 0 & 0 & 0 & 0 & 0 & 0 & 0 & 1 \\
\hline 4 & 4 & 1 & 0 & 0 & 0 & 0 & 0 & 0 & 9 \\
\hline 144 & 192 & 88 & 16 & 1 & 0 & 0 & 0 & 0 & 441 \\
\hline 14400 & 22080 & 12784 & 3552 & 508 & 36 & 1 & 0 & 0 & 53361 \\
\hline 2822400 & 4730880 & 3138304 & 1076224 & 211808 & 24832 & 1712 & 64 & 1 & 12006225 \\
\hline
\end{tabular}

Table 3. $0 \leq n, k \leq 4, r=2$, and $s=3$.

\begin{tabular}{ccccccccccccc}
\hline 1 & 0 & 0 & 0 & 0 & 0 & 0 & 0 & 0 & 0 & 0 & 0 & 1 \\
8 & 12 & 6 & 1 & 0 & 0 & 0 & 0 & 0 & 0 & 0 & 0 & 27 \\
1728 & 3456 & 2736 & 1088 & 228 & 24 & 1 & 0 & 0 & 0 & 0 & 0 & 9261 \\
1728000 & 3974400 & 3824640 & 2014208 & 639936 & 127776 & 16128 & 1248 & 54 & 1 & 0 & 0 & 12326391 \\
4741632000 & 11921817600 & 12904335360 & 7944527872 & 3104947968 & 515321088 & 148279040 & 18914304 & 1687152 & 103040 & 4104961 & 41601569625 \\
\hline
\end{tabular}

Notice that the last column in all tables is just the sum of the entries of the corresponding row. 